\title{
A New Method to Prove Goldbach's Conjecture
}

\author{
Zengyong Liang \\ MCHH of Guangxi, Nanning, China \\ Email:1zy8ok@163.com
}

How to cite this paper: Liang, Z.Y. (2022) A New Method to Prove Goldbach's Conjecture. Advances in Pure Mathematics, 12, 1-9.

https://doi.org/10.4236/apm.2022.121001

Received: December 17, 2021

Accepted: January 22, 2022

Published: January 25, 2022

Copyright ( 2022 by author(s) and Scientific Research Publishing Inc. This work is licensed under the Creative Commons Attribution International License (CC BY 4.0). http://creativecommons.org/licenses/by/4.0/

\begin{abstract}
This paper introduces how to use geometric figures to represent integers, and successfully proves Goldbach's conjecture by using the mapping relationship between the internal angles of circles and sectors and the number of integers. It is also explained and proved that $w(n)$ is the function that calculates the lower limit of the number of prime pairs. A very effective new method is found to solve this kind of integer problems.
\end{abstract}

\section{Keywords}

Mapping, Sector, Sieve, Component, Induction

\section{Preface}

\subsection{Definition of Mathematical Symbols}

In this paper:

- $A$ denotes the set of positive integers in the interval $[1,2 n]$ interval, that is, $A=\{1,2,3, \cdots, 2 n\}$, and the number of elements in set $A$ is recorded as $|(A)|$.

- $A_{P}$ is a subset containing multiples of prime $p$, i.e. $A_{p}=\{1 p, 2 p, 3 p, 4 p, 5 p, \cdots\}$

- $P$ represents the set of prime numbers, $p$ or $p_{i}$ represents the prime number, and $p_{m}$ represents the maximum prime number with not greater than $\sqrt{n}$.

- $\pi(n)$ is the number of prime numbers with not greater than $n$.

- $D(2 n)$ is the number of prime pairs of even $2 n$.

- $\phi(n)$ is Euler function.

- $\phi^{\prime}(n)$ is the lower bound function of the number of non composite numbers.

- $w(n)$ is a function for calculating the lower limit of the number of prime pairs.

\subsection{Completed Achievements}

The paper "Rigorous Proof of Goldbach's Conjecture" has obtained the follow- 
ing results for proving Goldbach's conjecture [1]:

$2 n$ positive integers are arranged by using the method of legend, as shown in Figure 1 . There are $2 n-1$ pairs of integers that sum of two integers equals $2 n$ (it is called integer pairs). If it can be proved that one of integer pairs is containing two primes (it is called prime pairs), the Goldbach's conjecture is proved [2].

According to the screening method, the lower limit of the number of primes $\pi(n)$ is obtained by using excessive screening, i.e.

$$
\pi(n) \geq \phi^{\prime}=n \prod_{i=1}^{m}\left(1-\frac{1}{p_{i}}\right)
$$

And using the over sieving method, the function $d(n)$ for calculating the lower limit of the number of prime pairs is obtained, which is

$$
D(2 n) \geq w(n)=\frac{n}{2} \prod_{i=2}^{m}\left(1-\frac{2}{p_{i}}\right)
$$

It is proved that when $w(n) \geq 4$, there must be one or more of prime pairs.

In addition, it is proved that

$$
w(n)=\frac{n}{2} \prod_{i=2}^{m}\left(1-\frac{2}{p_{i}}\right) \geq \frac{p_{m}}{4}
$$

Then, when $p_{m} \geq 17$ and $2 n \geq 290, \frac{p_{m}}{4}=4.25$, this proves that there must be one or more prime pairs. (The function $w(n)$ was defined and wrote by " $d(n)$ " in paper [1].)

This paper will continue the proof of Goldbach's conjecture using geometric figures.

\section{Geometry and Number}

\subsection{Relationship between Geometry and Number}

The most typical example of proving mathematical theorems with geometric figures is Pythagorean theorem [2] [3]. Pythagorean successfully proved Pythagorean theorem by using the corresponding relationship between the area and square number of right triangle. Now, we intend to prove Goldbach's conjecture by the mapping correspondence between circle and integers [3].

The relationship between rational numbers and integers can be expressed by integer points on the number axis (or line), see Figure 2.

1) Integer points on the number axis.

2) There are 9 integer points on a straight line with a length of 9.5. There are 11 integer points on a straight line with a length of 11.5.

We can use many sectors of a circle to represent a set of integers (integers with a region of $[1,2 n]$ ), as shown (1) in Figure 3. So, a small sector to represent

$$
\begin{array}{cccccccc}
1 & 2 & 3 & \ldots & n \ldots & 2 n-3 & 2 n-2 & 2 n-1 \\
2 n-1 & 2 n-2 & 2 n-3 & \ldots . & \ldots & 3 & 2 & 1
\end{array}
$$

Figure 1. $2 n-1$ integer pairs. 


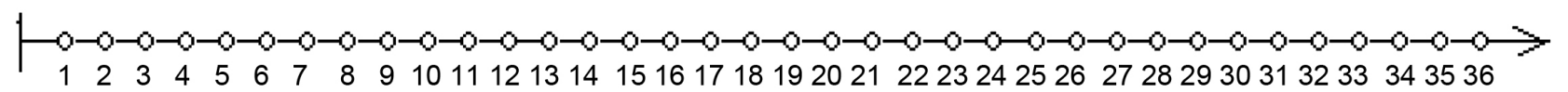

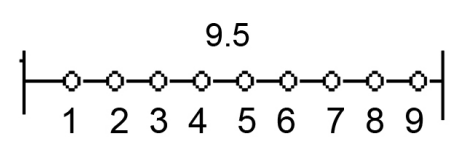

(2)
(1)

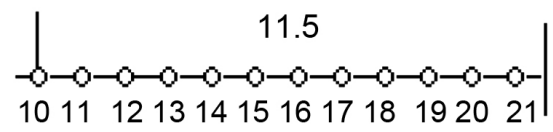

(3)

Figure 2. It is the relationship between rational numbers and integers.

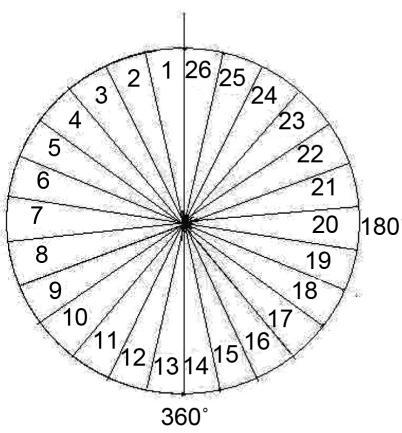

(1)

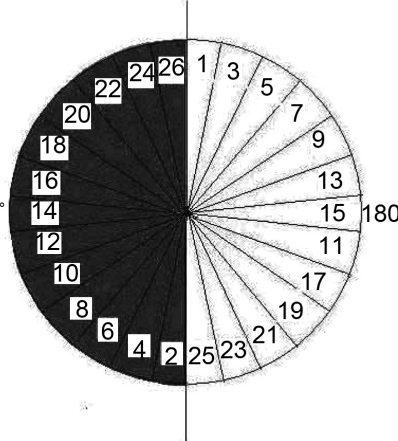

(2)

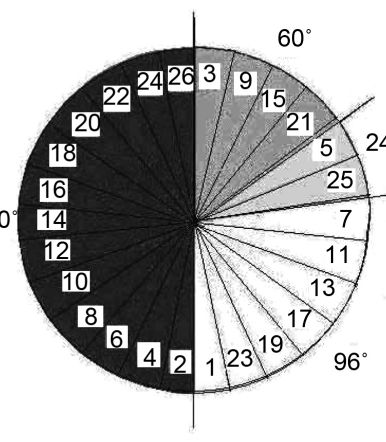

(3)

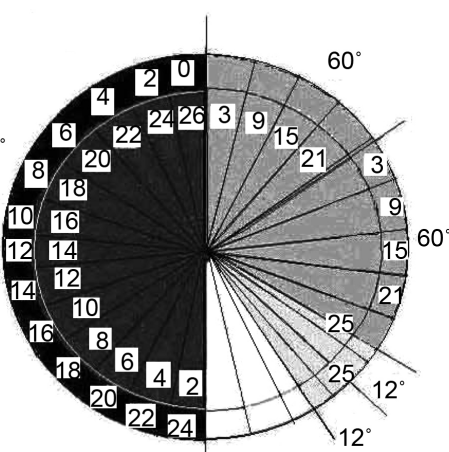

(4) 36

Figure 3. It is shown that of mapping relationship between circle and integers.

an integer, and interior angle of sector is $\theta(\theta=360 / 2 n)$. In addition, $2 n$ integers have a one-to-one integer mapping relationship with the internal angle of the sector. In this way, we can use geometry to understand the complex relationship of various numbers and solve problems. For example, $2 n=26,26$ small sectors represent 26 integers, let $\gamma$ Is its internal angle of a small sector, then $\gamma=360 / 26 \approx 13.846^{\circ}$.

Let set of $2 n$ integers be $A$, and set of small sectors be $S$, set $A$ has a one-to-one surjection relationship with set $S$, i.e., $A \Leftrightarrow S$. At the same time, $2 n$ integers have a one-to-one integer mapping relationship with the circumference (arc length) and the interior angle (or area) of the sector in the circle [4]. $n$ and $\theta$ The functional relationship is

$$
\theta=f(n), \theta=\frac{360}{2 n}, n>0 \text { and } n \in N
$$

Some definition:

- Interior angle of sector (or circle) is noted by $\theta^{\circ}$.

- A small sector represents an integer, and its interior angle is noted by $\gamma^{\circ}$, $\gamma=\frac{360}{2 n}$.

- An interior angle of sector with $\left|A_{p}\right|$ is noted by $\alpha_{p}, \alpha_{p}=\left|A_{p}\right| \times \gamma^{\circ}$.

- Component of $p$ in a sector (primitive or remaining) is noted by $\beta_{p}$. For example, if in a circle, $\beta_{p}=\frac{360}{p}$; in a semicircle, $\beta_{3}^{\prime}=\frac{180}{3}$; in a sector of $60^{\circ}$, 
$\beta_{5}^{\prime}=\frac{60}{5}$.

Theorem 1. Let an interior angle of sector with $\left|A_{p}\right|$ be $\alpha_{p}^{\circ}$, then $\alpha_{p} \leq \beta_{p}$. Proof. Because in $2 n$ integers, $\beta_{p}=\frac{360}{p},\left|A_{p}\right|=\left[\frac{2 n}{p}\right]$, $\alpha_{p}=\left[\frac{2 n}{p}\right] \times \gamma=\left[\frac{2 n}{p}\right] \times \frac{360}{2 n}:$

1) When $p \mid 2 n,\left[\frac{2 n}{p}\right]=\frac{2 n}{p}, \alpha_{p}=\left[\frac{2 n}{p}\right] \times \frac{360}{2 n}=\frac{2 n}{p} \times \frac{360}{2 n}=\frac{360}{p}$, then $\alpha_{p}=\beta_{p}$;

2) In addition, $p \nmid 2 n$, then $\left[\frac{2 n}{p}\right]<\frac{2 n}{p}, \alpha_{p}=\left[\frac{2 n}{p}\right] \times \frac{360}{2 n}<\frac{2 n}{p} \times \frac{360}{2 n}=\frac{360}{p}$, so $\alpha_{p}<\beta_{p}$.

More generally, $\left[\frac{n}{p}\right] \times \frac{180}{n}<\frac{n}{p} \times \frac{180}{n}$, so $\alpha_{p}^{\prime}<\beta_{p}^{\prime}$.

From Theorem 1, we get a very important principle. However large the $2 n$ (including small sectors) is, the components $\beta_{p}$ are the same $\left(\frac{\theta}{p}\right)$. This is very helpful to prove. When we need to delete multiples of prime $p$, just delete the corresponding sector of $\beta_{p}$ (the corresponding multiples of $p$ can be completely screened out). At the same time, no matter how big the $\left|A_{p}\right|$ is, the form of formula is the same.

\subsection{Sieve Method}

\subsubsection{Sieving of Composites}

Therefore, to screen out the composites of primes $2,3,5, \cdots, p_{m}$ in $2 n$ by using method of the "sieve of Eratosthenes", it is only necessary to delete the component of each prime number to $2 n$, and according to the following steps [5]. The specific operations are as follows:

1) First, we delete the even component $\beta_{2}=360 / 2$ (paint the semicircle representing even number with black), which the remaining is $180^{\circ}$. The semicircle (white) of represents the odd component, see (2) in Figure 3

$$
f(2 n)=360\left(1-\frac{1}{2}\right)=360-180=180
$$

2) Delete this integer pairs of multiples of 3 by deleting a sector of $\beta_{3}^{\prime}$ (painted dark grey) in the sector of $180^{\circ}$. Since $\beta_{3}^{\prime}=\frac{180}{3}=60^{\circ}$, then

$$
f(2 n)=360\left(1-\frac{1}{2}\right)\left(1-\frac{1}{3}\right)=180-\frac{180}{3}=120 \text {. }
$$

3) Delete component of 5 by deleting a sector of $\beta_{5}^{\prime}$ (painted light gray) in the sector of $120^{\circ}$. Since $\beta_{5}^{\prime}=\frac{120}{5}=24^{\circ}, f(n)$ corresponding to this 
operation is

$$
f(2 n)=360\left(1-\frac{1}{2}\right)\left(1-\frac{1}{3}\right)\left(1-\frac{1}{5}\right)=360-180-60-24=96,
$$

see (3) in Figure 3.

And so on $\cdots$, finally get

$$
f(2 n)=360\left(1-\frac{1}{2}\right)\left(1-\frac{1}{3}\right)\left(1-\frac{1}{5}\right) \cdots\left(1-\frac{1}{p_{m}}\right) .
$$

Refer to Figure 3 for this operation. This specific method is as follows:

1) 26 integers (or pairs of integers) are represented by a circle. Each interior angle of sector is $\gamma^{\circ}$. A small sector represents an integer. $\gamma=360 / 26=13.846^{\circ}$.

2) Even and odd numbers account for 180 respectively, which are two semicircles (or even and odd pairs).

3) There are 4 multiples of $3, \alpha_{3}=4 \gamma=4 \times 13.846=55.384^{\circ}$, it is less than $\beta_{3}^{\prime}=60^{\circ}$. Deleted multiples of 3 (by $\beta_{3}^{\prime}$ ), interior angle of remaining sector is $x^{\circ}, \quad x=180-60=120^{\circ} . \beta_{5}^{\prime}=120 / 5=24^{\circ}$. Then, we delete component of 5 (by $\beta_{5}^{\prime}$ ) finally get a remaining sector, its interior angle is $120^{\circ}-24^{\circ}=96^{\circ}$. There are 6 of multiples of 3 and $5 . \alpha_{3}+\alpha_{5}=6 \gamma=6 \times 13.846=83.076^{\circ}$, it is less than $\beta_{3}^{\prime}+\beta_{5}^{\prime}=60+24=84^{\circ}$. When is deleted component of $\beta_{3}^{\prime}$ and $\beta_{5}^{\prime}$, there's still left $96^{\circ}$ of. interior angle remaining sector (white). Since

$96 / \gamma=96 / 13.846=6.933$. It is shown that there are at least 6 complete integers, excluding integer 1 , there are at least 5 odd primes.

\subsubsection{Sieving of Integer Pairs}

The following is the case of using graphics to screen out integer pairs. Now the circle represents $2 n$ integer pairs.

Theorem 2. When $2 n$ is a multiple of odd prime $p$, all multiples of $p$ are relative; when $2 n$ is not a multiple of prime $p$, all multiples of $p$ are not relative.

Proof. When $2 n$ is a multiple of odd prime $p$, that is, $n$ is a multiple of prime $p$, and $k p$ away from $n$ is also a multiple of $p$. So it is that all multiples of $p$ with above and below are relative. Conversely, when $2 n$ is not a multiple of prime $p$, all multiples of $p$ are not relative.

From Theorem 2, it can be seen that when $2 n$ is not a multiple of prime $p$, all integer pairs containing odd multiples of $p$ are not relative, which is the worst case for formation of prime pairs. In this way, when we discuss the elimination of integer pairs with composite numbers, we take the latter case which is most unfavorable to the generation of prime pairs as the condition. When we screen integer pairs with composite numbers, we screen out integer pairs with odd multiples of pat the top and bottom once at the same time. The formula is as follows:

$$
g(2 n)=360\left(1-\frac{1}{2}\right)\left(1-\frac{2}{3}\right)\left(1-\frac{2}{5}\right) \cdots\left(1-\frac{2}{p_{m}}\right) .
$$

The specific methods are as follows: 
1) First, we delete even pairs by deleting a semicircle of $\beta_{2}$.

$$
g(n)=360-180=180 .
$$

2) Delete this integer pairs of multiples of 3 by $\beta_{3}^{\prime}$ in semicircle on the right, $2 \times \beta_{3}^{\prime}=2 \times \frac{180}{3}=120$, as

$$
g(n)=360-180-120=180
$$

or

$$
g(2 n)=360\left(1-\frac{1}{2}\right)\left(1-\frac{2}{3}\right)=180-120=60 .
$$

3) Similarly, screen out the composites of 5 in the remaining sector $\left(60^{\circ}\right)$ (painted light gray) $\beta_{5}^{\prime}=\frac{60}{5}=12 . \theta=2 \times 12=24$. Finally, the remaining interior angle of sector is $36^{\circ}$. See (4) in Figure 2. The $g(n)$ corresponding to this operation is

$$
g(2 n)=360\left(1-\frac{1}{2}\right)\left(1-\frac{2}{3}\right)\left(1-\frac{2}{5}\right)=180-2 \times 60-2 \times 12=36 .
$$

And so on $\cdots$, finally get

$$
g(2 n)=360\left(1-\frac{1}{2}\right)\left(1-\frac{2}{3}\right)\left(1-\frac{2}{5}\right) \cdots\left(1-\frac{2}{p_{m}}\right) .
$$

Refer to Figure 2 for this operation, Figure 3 is shows that:

1) 26 integers (or pairs of integers) are represented by a circle. Each small sector represents an integer pair, which a interior angle is $\gamma, \gamma=360 / 26=13.846$.

2) Even and odd numbers account for $180^{\circ}$ respectively, which are two semicircles (or even and odd pairs).

3) There are the two circle represents two set of 26 integers. Use excessive screening is $g(n)=180-2 \times 60-2 \times 12=36^{\circ}$. Multiples of 3 and composites of 5 are marked here. (it is painted black and light gray respectively, and indicates that it is deleted). The last remaining sector (inner angle is $36^{\circ}$ ). Since $36 / \gamma=36 / 13.846=2.6$. it is that, there are remaining of at least two integer pairs.

In this way, however large $2 n$ is, according to Theorem 1 , we can use the same method of screening to screen out the composites of prime numbers, and finally get the lower limit of the number of prime numbers (or the number of prime pairs), as shown in Figure 4. At the same time, we get the functions $f(2 n)$ and $g(n)$ as follows

$$
\begin{aligned}
& f(2 n)=360 \prod_{i=1}^{m}\left(1-\frac{1}{p_{m}}\right) \\
& g(n)=180 \prod_{i=2}^{m}\left(1-\frac{2}{p_{m}}\right)
\end{aligned}
$$




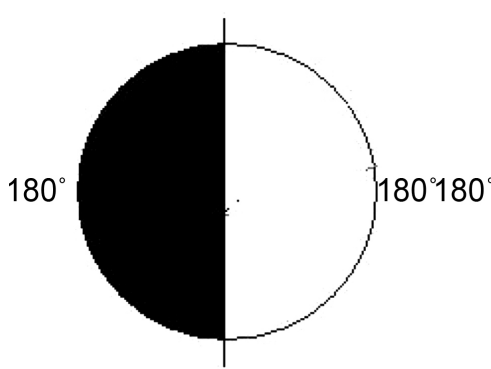

(1)

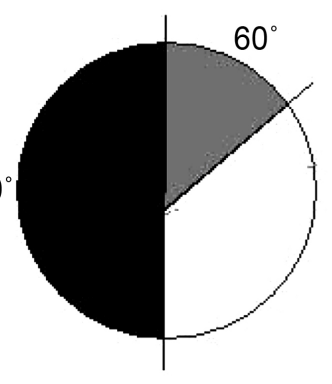

(2)

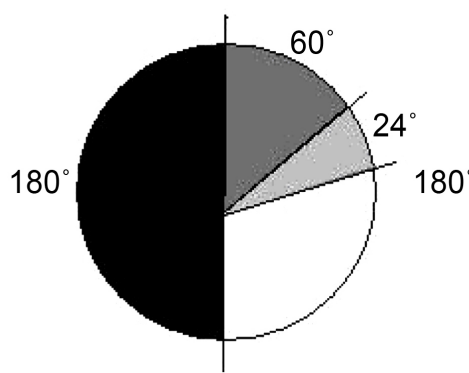

(3)

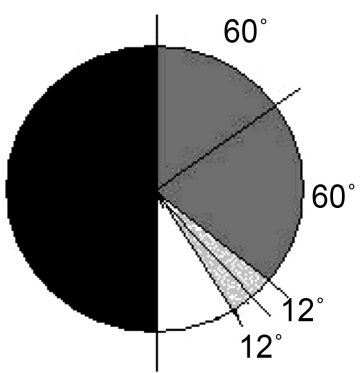

(4)

Figure 4. The process of deleting component of composites or integer pairs containing.

\section{Prove of Proposition}

Proposition: any even number greater than 4 can be expressed as the sum of two prime numbers.

The proof is known from subsections 1 and 2. If it can be proved that after over screening all composite numbers, there are at least one or more prime pairs in the last remaining sector, the proposition can be proved.

Next, we use mathematical induction for $\mathrm{m}$ to prove [6]:

1) When $p_{m}=7,2 n=7^{2}+1, \gamma=360 / 50=7.2^{\circ}$, deleted the components of all composites, if the last remaining internal angle of the sector is $\alpha^{\circ}$, $\alpha^{\circ}=180-2 \times 60-2 \times 12-2 \times 5.14=25.72^{\circ}, \quad 25.72 / 7.2 \approx 3.572$, namely $\alpha / \gamma=\alpha /\left[360 /\left(p_{7}^{2}+1\right)\right] \geq 3$.

2) When $m=k, 2 n=p_{k}^{2}+1$, here, $\gamma^{\prime}=360 /\left(p_{k}^{2}+1\right)$. Suppose that the last remaining internal angle of the sector is $x^{\circ}$ and $x / \gamma^{\prime} \geq 3$. Namely

$$
x / \gamma^{\prime}=x /\left[360 /\left(p_{k}^{2}+1\right)\right]=x\left(p_{k}^{2}+1\right) / 360 \geq 3 .
$$

So, when $m=k+1,2 n=p_{k+1}^{2}+1$, and $\gamma^{\prime \prime}=360 /\left(p_{k+1}^{2}+1\right)$, let that the last remaining internal angle of the sector is $y^{\circ}$, we have

$$
y / \gamma^{\prime \prime}=y /\left[360 /\left(p_{k+1}^{2}+1\right)\right]
$$

Because $\beta_{k+1}^{\prime}=x / p_{k+1}$,

$$
y=x-2 \beta_{k+1}^{\prime}=x-2 x / p_{k+1}=x\left(1-2 / p_{k+1}\right),
$$

By (10) and (11) get

$$
\begin{aligned}
y / \gamma^{\prime \prime} & =y /\left[360 /\left(p_{k+1}^{2}+1\right)\right] \\
& =\left[x^{\circ}\left(1-2 / p_{k+1}\right)\right] /\left[360 /\left(p_{k+1}^{2}+1\right)\right] \\
& =x\left(p_{k+1}^{2}+1\right)\left(1-2 / p_{k+1}\right) / 360 \\
& =x\left(p_{k+1}^{2}+1\right)\left(p_{k+1}-2\right) / p_{k+1} / 360 \\
& >x p_{k+1}^{2}\left(p_{k+1}-2\right) / p_{k+1} / 360 \\
& >x p_{k+1}\left(p_{k+1}-2\right) / 360
\end{aligned}
$$

Let $p_{k+1}=p_{k}+d, d \geq 2$, then 


$$
\begin{gathered}
x p_{k+1}\left(p_{k+1}-2\right) / 360=x\left(p_{k}+d\right)\left(p_{k}+d-2\right) / 360, \\
x p_{k+1}\left(p_{k+1}-2\right) / 360 \geq x\left(p_{k}^{2}+1\right) / 360 .
\end{gathered}
$$

In this way, by (9), (12) and (13), we get

$$
y / \gamma^{\prime \prime}>x p_{k+1}\left(p_{k+1}-2\right) / 360 \geq x\left(p_{k}^{2}+1\right) / 360 \geq 3
$$

Because the remaining sector may be contain two integer pairs containing 1 $(\{1,2 n-1\},\{2 n-1,1\})$, the other integer pair must be a prime pair. Namely there is least one of prime pair.

Now, we have been proved the case when $2 n=p_{m}^{2}+1$. For $2 n$ by the case from $p_{m}^{2}+1$ to $p_{m+1}^{2}+1$ is also valid because $m$ remains unchanged but $2 n$ increases. Therefore, when $2 n$ is greater than 50 , we can prove it graphically Goldbach's conjecture holds. (As for the solution of $2 n$ in $[4,48]$, it is easy to prove by list.)

\section{Another Proof}

If we make the circumference of a circle equal to $2 n$, an arc with an arc length of 1 corresponds to an integer.

We can use $2 n$ instead of 360 In this way, it can be derived from formula (4) and (5) as

$$
\begin{gathered}
f(2 n)=360 \coprod_{i=1}^{m}\left(1-\frac{1}{p_{i}}\right) \Leftrightarrow \phi^{\prime}(2 n)=2 n \coprod_{i=1}^{m}\left(1-\frac{1}{p_{i}}\right) \\
g(n)=180 \coprod_{i=2}^{m}\left(1-\frac{2}{p_{i}}\right) \Leftrightarrow w(n)=\frac{n}{2} \coprod_{i=2}^{m}\left(1-\frac{2}{p_{i}}\right) .
\end{gathered}
$$

So that we can prove that functions of $\phi^{\prime}(n)$ and $d(n)$ is right. At the some time, we can use formulas of those functions to prove Goldbach's conjecture.

It is worth mentioning that by changing the integer pair in Figure 1 into each integer pairs with two integers that difference of 2 (for example, $\{1,3\},\{2,4\},\{3$, $5\}, \cdots,\{2 n, 2 n+2\})$, by $w(n) \geq \frac{p_{m}}{4}$, and $p_{m} \rightarrow \infty \rightarrow w(n) \rightarrow \infty$, we can easily prove another world mathematical problem that about infinite twin prime numbers. This is a very gratifying thing.

\section{Conclusion}

Mathematics and geometry are closely related. Geometric figures are used to represent the relationship between numbers. Because the quantity of geometric figures has a one-to-one mapping relationship with rational numbers, it obviously shows the relationship between prime numbers and composite numbers in natural numbers, the relationship between rational numbers and integers, and the results of screening the changes of operands. A complicated mathematical problem in the world that has not been solved for hundreds of years has been solved rigorously. This is the charm of morphological mathematics. It can be 
said that this proof is simple and easy to understand. The theory of elementary mathematics used in the proof does not seem to be complex [7]. It is a simple, clear and rigorous proof of Goldbach's conjecture.

\section{Conflicts of Interest}

The author declares no conflicts of interest regarding the publication of this paper.

\section{References}

[1] Liang, Z.Y. (2018) Rigorous Proof of Goldbach's Conjecture. Journal of Applied Mathematics and Physics, 6.

[2] Euclid (2011) Geometric Origin. Trans. by Yan X.D. Jiangsu People’s Publishing House, Nanjing, 44.

[3] Apostol, T.M. (2016) Introduction to Analytic Number Theory. Trans. by Tang T.M. Harbin Institute of Technology University Press, Harbin, 1-2.

[4] Department of Applied Mathematics of Tongji University (2008) Higher Mathematics. Higher Education Press, Jinan, 1-2.

[5] Rosen, K.H. (2005) Elementary Number Theory and Its Applications. China Machine Press, Beijing, 18-19.

[6] Harly, G.H. and Wright, E.M. (2007) An Introduction to the Theory of Numbers. Posts \& Telecom Press, Beijing, 3-4.

[7] Baylor, A.H. (2015) Recreations in the Theory of Numbers-The Queen Mathematics Entertains. Trans. by Dan X.B. Shanghai Education Press, Shanghai, 306-312. 